\begin{tabular}{|c|c|}
\hline \multicolumn{3}{|c|}{$\begin{array}{c}\text { Indian Journal of Medical Sciences } \\
\text { (INCORPORATING THE MEDICAL BULLETIN) }\end{array}$} \\
\hline VOLUME 60 & APRIL 2006 \\
\hline \multicolumn{3}{|c|}{ ORIGINAL CONTRIBUTIONS } \\
\hline
\end{tabular}

\title{
VARIATIONS IN THE CEREBRAL VENOUS ANATOMY AND PITFALLS IN THE DIAGNOSIS OF CEREBRAL VENOUS SINUS THROMBOSIS: LOW FIELD MR EXPERIENCE
}

\author{
N. R. S. SURENDRABABU, SUBATHIRA, R. S. LIVINGSTONE
}

\section{ABSTRACT}

BACKGROUND: Knowledge of variations in the cerebral venous anatomy and apparent signal abnormalities seen on Magnetic resonance (MR) angiography are essential to avoid over-diagnosis of cerebral venous sinus thrombosis (CVST), while interpreting the MR angiograms. AIMS: To evaluate the variations of cerebral venous anatomy and signal abnormalities by using 3D phase contrast MR angiography performed in a 0.5 Tesla MRI scanner. MATERIALS AND METHODS: One hundred patients who underwent $M R$ imaging and $M R$ angiography examinations at our institution from March 2004 to February 2005, with normal MR imaging of brain were studied retrospectively. Patients with clinical suspicion of CVST and patients who underwent color doppler evaluation for suspected deep vein thrombosis were excluded. RESULTS: The superior sagittal, straight sinus and the internal cerebral veins were visualized in all patients. There was hypoplasia of the right transverse sinus in 13 patients, left transverse sinus in 35 patients, right sigmoid sinus in 6 patients and left sigmoid sinus in 19 patients. Absence of transverse sinus on left side was observed in one patient and absence of sigmoid sinus in 2 patients on left side. Flow gaps were observed in non-dominant transverse sinus, sigmoid sinus as well as transverse sigmoid sinus junctions. The occipital sinus was visualized in 17 patients. CONCLUSIONS: MR angiography done at low field strengths is also a reliable method, for assessing cerebral venous sinuses. Awareness of the normal anatomical variations of venous sinuses and apparent MR angiographic flow gaps prevent misdiagnosis of cerebral venous sinus thrombosis.

Key words: MR angiography, cerebral venous anatomy, cerebral venous sinus thrombosis, cerebral venous thrombosis, Low field MR.

Department of Radiodiagnosis, Christian Medical College and Hospital, Vellore - 632004 , India

\section{Correspondence}

Dr. Narayanam R. S. Surendrabab

Department of Radiodiagnosis, Christian Medical College and Hospital, Vellore - 632 004, Tamilnadu, India.

\section{INTRODUCTION}

Magnetic resonance (MR) angiography is one of the accepted methods of evaluation of the cerebral venous sinus anatomy as well as pathology..$^{[1,2]}$ Among others, the CT venography and conventional angiography are notable methods. The disadvantages of the conventional angiography are the use of ionizing radiation and the invasiveness of the procedure with its inherent complications. The CT Venography also involves high radiation dose. However, the use of MR angiography has got several advantages compared to other methods. In the MR angiography, there is a possibility of acquiring images with or without the use of contrast media. Techniques used to perform the MR angiography without the injection of gadolinium chelete are, the time-of-flight (TOF) and phase-contrast (PC) angiography. Two dimensional (2D) and three dimensional (3D) acquisitions can be used in either of these techniques. There are many pitfalls in the diagnosis of the cerebral venous sinus thrombosis (CVST) on MR angiography. The study aims to evaluate the normal MR appearances of cerebral venous sinus anatomy and its variations, as well as the apparent MR signal abnormalities seen normally, in the MR angiography done using a 0.5 Tesla MR equipment.

\section{MATERIALS AND METHODS}

All the MR examinations were performed using a 0.5T MR equipment (Philips Gyroscan T5-NT, Netherlands) in our institution where the study was conducted. The MR angiographic examinations were performed using the 3D phase contrast technique without applying the inferior saturation band. The slice thickness of 1.2 $\mathrm{mm}$ was acquired with contiguous sections using matrix size of $256 \times 256$ and a nominal field of view (FOV) of $27 \mathrm{~cm}$ with TR (Time to Repeat) -22 milliseconds, TE (Time to Echo) -7.4 milliseconds and flip angle of $15^{\circ}$. The velocity encoding applied was $30-40 \mathrm{~cm}$ per second. The images were displayed as 20 maximum intensity projection (MIP) images reconstructed from the source images at 9 degree increments.

Patients who underwent MR imaging at our institution were selected from picture archiving and communication system (PACS) database, from March 2004 to February 2005. One hundred patients were selected by viewing the normal MR imaging of the brain, who also had MR angiographic examination. The age of patients included was between 2 to 68 years with a median age of 35 years. These patients MR angiograms were systematically reviewed in the PACS (General Electric, Milwaukee, USA) workstation using high resolution monitors.

The dural venous sinuses included in this study are superior sagittal sinus, sigmoid sinus, transverse sinus, straight sinus and occipital sinus. Other major cerebral veins which were relatively constant and included in the study were internal cerebral veins, superficial cortical veins i.e, veins of Trolard and Labbe. The clinical indication of the headache was also included in the study as it was a general symptom for which clinicians would require cross sectional imaging in order to rule out any other intracranial 
abnormalities. Twenty six patients with this indication were included in our study with normal MR imaging. All these twenty six patients were followed up clinically for a period of 6 months, who had treated with antimigraine therapy and all of them improved with this therapy. Patients with ischemic changes, lacunar infarcts, tumors, myelination disorders, small vessel disease and other congenital and acquired morphological abnormalities were excluded from the study. Patients with clinical indication of cerebral venous sinus thrombosis (CVST) and clinical suspicion of deep vein thrombosis (DVT) with color doppler imaging were also excluded from the study.

The transverse sinuses were measured $1 \mathrm{~cm}$ from the Torcula heterophili and the sigmoid sinuses were measured $1 \mathrm{~cm}$ from the transverse sigmoid junctions. These were compared with the superior sagittal sinus. If the linear measurement was less than half the size of the superior sagittal sinus, it was considered hypoplastic and if not visualised it was considered aplastic or absence of sinus. The internal jugular veins were not included in the study. The dominance of sinuses and variations in drainage of the superior sagittal sinus and straight sinus as well as flow gaps in these sinuses were studied.

\section{RESULTS}

Out of the one hundred patients studied, superior sagittal sinus, straight sinus and internal cerebral veins were seen in all cases [Figure 1]. Right transverse sinus was dominant in 59 patients, left transverse sinus was dominant in 30 patients and co-dominant sinuses were seen in 10 patients. The transverse sinus was hypoplastic [Figure 2] in 13 patients and 35 patients on right and left respectively. The transverse sinus was not seen on left side in one patient alone. Similarly, sigmoid sinus was hypoplastic [Figure 3], in 6 patients on right side and 19 patients on the left side. Two patients had aplastic sigmoid sinus on the left side.

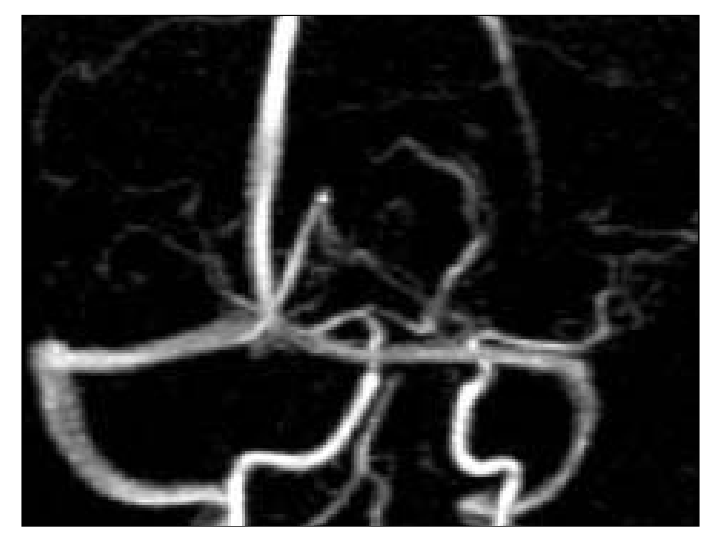

Figure 1: MR angiogram showing bilateral codominant transverse sinuses with visualization superior sagittal sinus, straight sinus and interna cerebral veins.

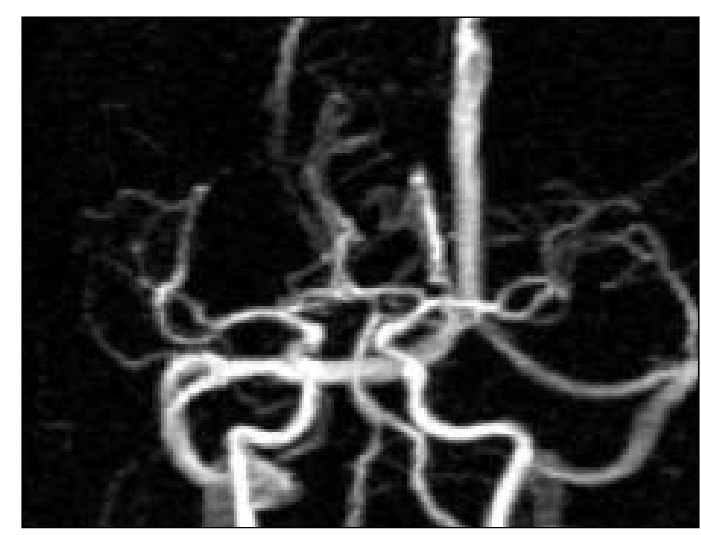

Figure 2: MR angiogram showing hypoplastic left transverse sinus with partial split right transverse sinus and arachnoid granulations causing flow gap in the superior sagittal sinus
The relatively constant superficial cerebral vein identified in our study was vein of Trolard, observed in 79 patients on right and 75 patients on left. Similarly the Vein of Labbe was found in 70 patients on right and 68 patients on left. The occipital sinus was identified in 17 patients in our study [Figure 4]. Majority of patients to the extent of $39 \%$, the superior sagittal sinus drained into the right transverse sinus, $15 \%$ drained into the left transverse sinus and remaining into the torcula. Contrary to the superior sagittal

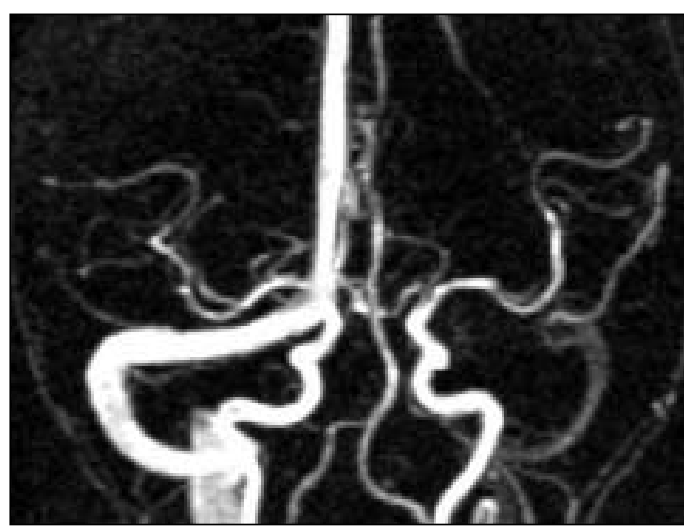

Figure 3: MR angiogram showing aplastic left transverse sinus, hypoplastic left sigmoid sinus with transverse sinus, hypop
prominent vein of labbe

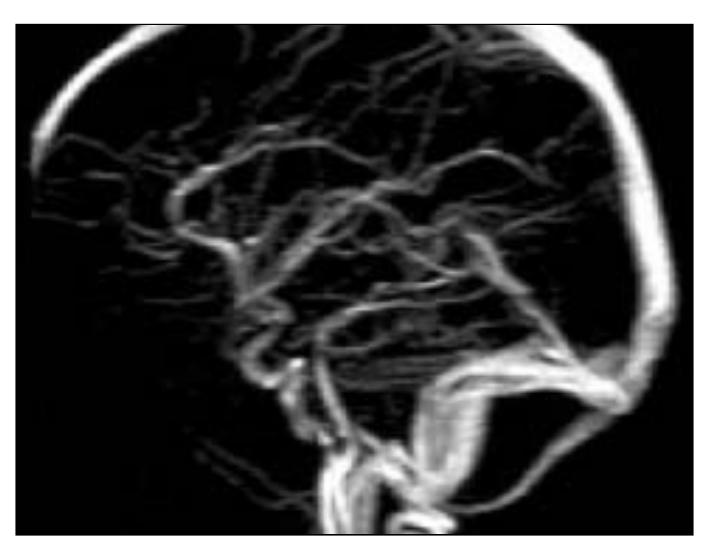

Figure 4: MR angiogram showing occipital sinus and flow gaps in the posterior part of superior sagittal sinus, right transverse and sigmoid sinus sinus, the predominant drainage of the straight sinus was to the left transverse sinus $(21 \%)$, to a lesser extent to the right transverse sinus (13\%) and remaining into the torcula. The flow gaps were observed in the transverse, sigmoid sinuses and in the transverse-sigmoid sinus junctions shown in [Table 1] [Figures 5, 6]. Superior sagittal sinus abnormalities are shown in [Table 2] and incidence of partial split sinus are shown in [Table 3], [Figure 7].

\section{DISCUSSION}

The incidence of the dural venous sinus thrombosis was $9.3 \%$ in a study done on autopsy series. ${ }^{[3]}$ The triad of symptoms for the cerebral venous sinus thrombosis are headache, seizures and loss of consciousness. ${ }^{[4]}$ Since these symptoms may vary, clinicians have to depend on the ancillary investigations. The common causes of this condition are dehydration, use oral contraceptives, cardiac disease with any infection and malignancy. Birth trauma, inflammation and malnutrition are important causes in children. Many a times the venous sinus thrombosis does not proceed further to Table 1: Flow gaps observed in transverse-sigmoid sinus junctions

\begin{tabular}{llcc}
\hline Flow gaps & Right & Left & Bilateral \\
\hline $\begin{array}{l}\text { Transverse sigmoid } \\
\text { sinus junctions }\end{array}$ & 15 & 19 & 11 \\
$\begin{array}{l}\text { Transverse sinus on } \\
\text { non-dominant side }\end{array}$ & 3 & 5 & - \\
$\begin{array}{l}\text { Transverse sinus on } \\
\text { dominant side }\end{array}$ & 2 & 1 & - \\
$\begin{array}{l}\text { Transverse sinus } \\
\text { co-dominant }\end{array}$ & 0 & 0 & 4 \\
$\begin{array}{l}\text { Sigmoid sinus on } \\
\text { non-dominant side }\end{array}$ & 3 & 4 & - \\
$\begin{array}{l}\text { Sigmoid sinus on } \\
\text { dominant side }\end{array}$ & 2 & 1 & - \\
$\begin{array}{l}\text { Sigmoid sinus } \\
\text { co-dominant }\end{array}$ & 1 & 0 & 2 \\
\hline
\end{tabular}


Table 2: Superior sagittal sinus

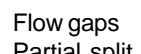

Partial split sinus

Non visuliased anterior $1 / 3$ of sinus 24
12
9

\section{Table 3: Partial split sinus}

Superior sagittal sinus

Transverse sinus

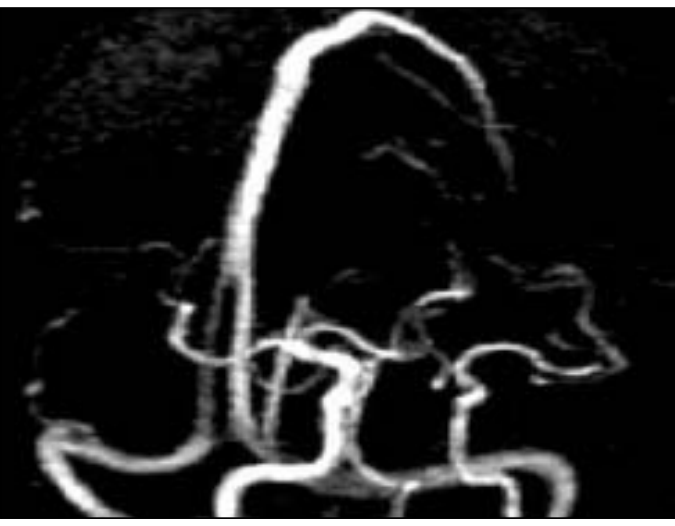

Figure 7: MR angiogram showing partial split superior sagittal sinus in the posterior aspect with flow gap in the right transverse sinus

vein thrombosis may result in early development of the venous infarctions with hemorrhagic transformation. ${ }^{[5,6]}$

MR angiography has proved to be one of the best methods of demonstrating venous sinus anatomy and venous sinus thrombosis replacing the conventional angiography in this aspect. ${ }^{[7,8]}$ Major cerebral venous sinuses are often studied by using the two dimensional time-of-flight MR angiography (2D TOF MRA) technique of MR angiography. ${ }^{[8,9]}$ Liauw et al, ${ }^{[9]}$ claimed that three dimensional Phasecontrast MR angiography (3D PC MRA) and 2D TOF MRA techniques of are equally good for visualization of the intracranial venous sinuses. PC MRA is very sensitive in cases of subacute dural sinus thrombosis where T1W hyperintensity is falsely suggested as patent sinus, in the 2D TOF technique. ${ }^{[10]}$ Usually high signal intensity is seen in the visualised veins in MRA, but absence of flow causes flow gap or absence of high signal.

Figure 6: MR angiogram showing a flow gap in the left transverse sinus, due to arachnoid granulation and flow gap in the medial aspect of the non-dominant transverse sinus Contrary to other studies which were done on 1.5T units using the 2D TOF technique, our study is based on the 3D PC MRA performed using $0.5 \mathrm{~T}$ equipment. The PC MRA has advantage of distinguishing the thrombus from flow, in contrast to the 2D TOF MRA It also has the advantage of excellent background suppression. Disadvantages are long imaging time and requires prior estimate of blood flow velocity. In addition to the anatomic variations, the flow gaps described also pose diagnostic difficulties in interpreting the MR angiograms.

In a study reported by Ayanzen et al, ${ }^{[11]}$ thirty-one percent of their patient population had flow gaps in the transverse sinus, 90\% of which were in the non-dominant sinus which are potentially indistinguishable from the thrombosis. Alper et al, ${ }^{[12]}$ described flow gaps in $24 \%$ of normal population. Our study revealed flow gaps [Table 1] more at the sigmoid transverse junctions or in the lateral most aspect of the transverse sinus and to a lesser extent in the sigmoid sinus. Flow gaps were not observed in the dominant side of the transverse and sigmoid sinuses in our study.

The PC MRA generates signal contrast between flowing and stationary nuclei by sensitizing the phase of the transverse magnetization to the velocity of motion. It has the capability for a limited range of velocities and is further limited by gradient imperfection and eddy currents. ${ }^{[13]}$ It can he effectively used to avoid problems of magnetization saturation that occur in 3D TOF studies. As discussed by Kirchhof et al, ${ }^{[14]}$ PC MRA can yield additional information about blood flow directions and absolute flow velocities. Inappropriate settings can lead to aliasing effects in which flow directionality is incorrectly displayed or reducing the sensitivity. Phase-contrast methods also impose strong demands on magnetic field gradient performance. ${ }^{[15]}$ The major drawbacks of phase contrast methods are the relatively lengthy acquisition times and the need to match the imaging gradient sensitivity to the anticipated flow conditions a priori. The PC MRA may also be more sensitive to signal loss due to turbulence, spin saturation and intravoxel dephasing. ${ }^{[16]}$ Apart from this reconstructions from MIP also suffer from its own limitations, often generating vascular images that exhibit intrinsic underestimation of vessel stenosis and flow gaps. ${ }^{[11]}$ However, these flow gap artifacts may be due to the intrinsic behavior of the nuclei dephasing leading to a loss of signal. These artifacts can be overcome by using appropriate settings (optimal imaging parameters) during acquisition.

Alper et al, ${ }^{[12]}$ described about transverse sinus abnormalities - $20 \%$ aplasia and $39 \%$ hypoplasia of the left sinus, $6 \%$ hypoplasia and $4 \%$ aplasia of the right sinus and $31 \%$ symmetric sinuses. Our results also show most of the hypoplastic and aplastic transverse sinuses were on the left side only. During fetal development as the volume of the telencepahlon increases, the torcula descends resulting in the inferior inclination of the lateral portion of the transverse sinuses which becomes less marked. During the period of transition from the stage of plexiform networks to that of regular transverse sinus there is rapid increase and then decreased in caliber occurs in the region of the torcula and the lateral sinus, may in 
some cases become irregular or the lateral sinus may be markedly hypoplastic or may even be absent in its major portion. Less commonly, the lateral portion of the transverse or sigmoid sinus may also be hypoplastic or absent. Unequal heights, asymmetrical sizes, mild to marked irregularities, or even absence of the medial portion of the transverse sinuses are therefore frequently encountered. ${ }^{[17]}$ Ruiz et $a l,{ }^{[18]}$ reported the presence of occipital sinus in one of 12 cadavers with an average age at time of death of 85 years. In a study by Widjaja et $a l,{ }^{[19]}$ occipital sinuses were reported in $18 \%$. In our study we identified occipital sinus in $17 \%$ of patients. The partial split sinus especially in the region of the posterior aspect of the superior sagittal sinus is known to cause the misdiagnosis of the thrombosis. Embryologically superior sagittal sinus formed from the marginal sinus on either side fuse in mid line to form superior sagittal plexus from which it develops failure of the partial fusion resulting in the partial split sinus. ${ }^{[20]}$ The arachnoid granulations contained within venous lacunae, are found protruding into the sinus along its course and may produce filling defects on imaging studies especially in superior sagittal sinus and transverse sinus regions, which is also a contributing factor for this flow gaps and account for potential pitfall for thrombus. ${ }^{[21]}$

\section{CONCLUSIONS}

MR angiography done at low field strength is also a reliable tool in cerebral venous sinus assessment. Flow gaps seen in the transverse sinus and in the transversesigmoid junctions should not be mistaken as thrombus in all cases, where there is no index of suspicion for venous sinus thrombosis. Awareness of the norma anatomical variations of venous sinuses and apparent MR angiographic flow gaps would prevent over-diagnosis of cerebral sinus venous thrombosis.

\section{REFERENCES}

1. Isensee C, Reul J, Thron A. Magnetic resonance imaging of thrombosed dural sinuses. Stroke 1994:25:29-34.

2. Rippe DJ, Boyko OB, Spritzer CE, Meisler WJ, Dumoulin CL, Souza SP, et al. Demonstration of dural sinus occlusion by the use of MR angiography. Am J Neuroradiol 1990;11:199-201.

3. Towbin A. The syndrome of latent cerebral venous thrombosis: Its frequency and relation to age and congestive heart failure. Stroke 1973;4:419-30.

4. Bousser MG, Chiras J, Bories J, Castaigne P. Cerebral venous thrombosis-a review of 38 cases. Stroke 1985;16:199-213.

5. Horowitz M, Purdy P, Unwin H, Carstens G $3^{\text {rd }}$ Greenlee R, Hise J, et al. Treatment of dural sinus thrombosis using selective catheterization and urokinase. Ann Neurol 1995;38:58-67.

6. Frerichs KU, Deckert M, Kempski O, Schurer L, Einhaupl K, Baethmann A. Cerebral sinus and venous thrombosis in rats induces long-term deficits in brain function and morphology - evidence for a cytotoxic genesis. J Cereb Blood Flow Metab 1994;14:289-300.

7. Chakeres DW, Schmalbrock P, Brogan M, Yuan $C$, Cohen L. Normal venous anatomy of the brain: Demonstration with gadopentetate dimeglumine in enhanced 3-D MR angiography. Am J Roentgeno 1991;156:161-72.

8. Mattle HP, Wentz KU, Edelman RR, Wallner B, Finn JP, Barnes $\mathrm{P}$, et al. Cerebral venography with MR. Radiology 1991;178:453-8.

9. Liauw L, van Buchem MA, Spilt A, de Bruine FT, van den Berg R, Hermans J, et al. MR angiography of the intracranial venous system. Radiology 2000;214:678-82.

10. Nadel L, Braun IF, Kraft KA, Fatouros PP, Laine FJ. Intracranial vascular abnormalities: value of MR phase imaging to distinguish thrombus from flowing blood. Am J Roentgenol 1991;156:373-80.

11. Ayanzen RH, Bird CR, Keller PJ, McCully FJ, Theobald MR, Heiserman JE. Cerebral MR venography: Normal anatomy and potential diagnostic pitfalls. Am J Neuroradiol 2000;21:74-8.

12. Alper F, Kantarci M, Dane S, Gumustekin K, Onbas $\mathrm{O}$, Durur I. Importance of anatomical asymmetries of transverse sinuses: An MR venographic study. Cerebrovasc Dis 2004;18:236-9.

13. Tsuruda JS, Shimakawa A, Pelc NJ, Saloner D. Dural sinus occlusion: evaluation with phasesensitive gradient-echo MR imaging. Am J Neuroradiol 1991:12:481-8.

14. Kirchhof K, Welzel T, Jansen O, Sartor K. More reliable noninvasive visualization of the cerebra veins and dural sinuses: Comparison of three MR angiographic techniques. Radiology 2002;224:804-10

15. Saloner D. An introduction to MR angiography.
Radio Graphics 1995;15:453-65.

16. Perl J, Turski PA, Masaryk TJ. MR angiography In: Atlas SW, editors. Magnetic resonance imaging of brain and spine, $2^{\text {nd }}$ ed. Lippincot-Raven: Philadelpha; 1996. p. 1548-9.

17. Okudera T, Huang YP Ohta T, Yokota A, Nakamura $Y$, Maehara F, et al. Development of posterior fossa dural sinuses, emissary veins and jugular bulb: Morphological and radiologic study. Am J Neuroradiol 1994;15:1871-83.

18. San Millan Ruiz D, Gailloud P, Rufenacht DA, Delavelle J, Henry F, Fasel JH. The craniocervical venous system in relation to cerebral venous drainage. Am J Neuroradiol 2002;23:1500-8.

19. Widjaja E, Griffiths PD. Intracranial MR venography in children: Normal anatomy and variations. Am J Neuroradiol 2004;25:1557-62.

20. Huang YP, Okudera T, Ohta T, Robbins A. Anatomic variations of the dural venous sinuses. In: Knapp JP, Schmideck HH, editors. The Cerebral Venous System and Its Disorders. Grune and Stratton: Orlando; 1984. p. 109-67.

21. Scott JN, Farb RI. Imaging and anatomy of the normal intracranial venous system. Neuroimag Clin North Am 2003;13:1-12. 